\title{
Avaliação de empresas start-up por Opções Reais: o caso do setor de biotecnologia
}

\author{
Real Option valuation of start-up companies: the case of the \\ biotechnology industry
}

\author{
Pedro Henrique da Fonseca Rodrigues ${ }^{1}$ \\ Vicente Antonio de Castro Ferreira ${ }^{2}$ \\ Celso Funcia Lemme ${ }^{2}$ \\ Luiz Eduardo Teixeira Brandão ${ }^{3}$
}

\begin{abstract}
Resumo: Este trabalho avaliou uma empresa start-up de biotecnologia à luz da teoria de Opções Reais. O objetivo principal foi averiguar a adequação deste modelo na avaliação de empresas inovadoras, sujeitas a grande risco, de forma a captar o valor das flexibilidades gerenciais presentes neste tipo de empreendimento. Para verificar a subavaliação provocada pelo método de Fluxo de Caixa Descontado ao desconsiderar o valor das opções, foi realizado um estudo de caso na empresa BioNatural, incubada no Polo BioRio de Biotecnologia, localizado no campus da Universidade Federal do Rio de Janeiro. Os resultados da avaliação financeira de um projeto de pesquisa específico indicaram uma subavaliação da ordem de $16 \%$. Os resultados reforçam a visão de que a Teoria de Opções Reais pode ser mais adequada para avaliar empreendimentos sujeitos a extrema incerteza, captando um valor não mensurado pelas técnicas tradicionais de avaliação de projetos.
\end{abstract}

Palavras-chave: Avaliação de projetos. Opções Reais. Biotecnologia. Empresas start-up.

\begin{abstract}
In this study a biotech start-up company was evaluated using the Real Options method. The main objective was to verify whether the Real Options is a better pricing method for tech or biotech companies that operate in an uncertain enviroment. In order to determine the undervaluation resulting from the Discounted Cash Flow method without taking the Real Options into account the case of BioNatural, a start-up biotech company incubated at the BioRio Biotecnology Park, was analized. The results indicate a $16 \%$ undervaluation, approximately, and they indicate that the real options approach may be more appropriate to evaluate projects subject to extreme uncertainty since it can determine a value that is not measured by traditional valuation project methods.
\end{abstract}

Keywords: Project valuation. Real options. Biotechnology. Start-up companies.

\section{Introdução e objetivo}

O estudo das decisões de investimento vem sendo de grande interesse tanto para o meio acadêmico quanto para o mundo empresarial há décadas. Estabelecer critérios objetivos para a alocação eficiente de recursos sempre foi um dos principais desafios dos investidores.

O Valor Presente Líquido (VPL), citado por Copeland e Antikarov (2002) como o paradigma central do processo decisório, é a técnica mais difundida entre analistas de investimentos e executivos de empresas responsáveis pela alocação orçamentária. O critério do VPL, porém, ao modelar uma realidade estática, acaba por ignorar o valor da flexibilidade gerencial em investimentos sujeitos a significativas incertezas
(DIXIT; PINDYCK, 1994; TRIGEORGIS, 1996; AMRAM; KULATILAKA, 1999).

A capacidade do gestor em adiar um investimento em uma nova planta, expandir ou contrair a escala de produção, escalonar investimentos ou abandonar uma pesquisa mal sucedida, por exemplo, adiciona valor ao empreendimento. A valoração destas flexibilidades requer o uso de métodos de apreçamento de opções, como a metodologia das opções reais.

As empresas do setor de biotecnologia se enquadram nesta categoria de ativos. Projetos de Pesquisa e Desenvolvimento se caracterizam por apresentar extrema incerteza, tanto de mercado quanto

\footnotetext{
${ }^{1}$ Bain \& Company Brazil, Inc., Rua Olimpíadas, 205, $12^{\circ}$ andar, CEP 04551-000, São Paulo, SP, Brasil, e-mail: pedrohenriquefr@gmail.com

2 Instituto COPPEAD de Administração, Universidade Federal do Rio de Janeiro - UFRJ, Rua Pascoal Lemme, 355 , Ilha do Fundão, CEP 21941-918, Rio de Janeiro, RJ, Brasil, e-mail: vicente@ coppead.ufrj.br; celso@ coppead.ufrj.br

${ }^{3}$ Departamento de Administração, Pontifícia Universidade Católica do Rio de Janeiro - PUC-Rio, Rua Marques de São Vicente, 225, Gávea, CEP 22451-900, Rio de Janeiro, RJ, Brasil, e-mail: brandao@iag.puc-rio.br
}

Recebido em 26/8/2011 — Aceito em 17/9/2012

Suporte financeiro: $\mathrm{CNPq}$. 
técnica, possuindo alta flexibilidade do ponto de vista orçamentário e permitindo revisão e realocação constante dos investimentos planejados em caso de insucesso em qualquer das fases da pesquisa. Projetos da área de biotecnologia, portanto, não podem ser avaliados de forma estática, devendo ser incorporadas ao modelo de avaliação financeira as diversas opções de abandono, licenciamento, descoberta de novos produtos e pesquisas e outras.

A grande incerteza relativa ao sucesso das pesquisas e, posteriormente, ao sucesso da nova droga no mercado (LOCH; BODE-GREUEL, 2001) dificulta a avaliação correta dessas empresas. Segundo Villiger e Bogdan (2006), muitos profissionais da indústria consideram impossível atribuir um valor ao desenvolvimento de uma determinada droga, dada a existência de inúmeras incertezas relativas à segurança, eficácia, regulação, mercado e competição. Bode-Greuel e Greuel (2005) lembram que buscar uma forma objetiva de avaliação econômica é importante tanto para o gestor de uma farmacêutica em sua tarefa de definir quais projetos priorizar quanto para balizar negociações de parceria e licenciamento.

Neste trabalho analisamos o caso da empresa BioNatural (o nome real da empresa foi omitido a fim de preservar a confidencialidade das informações), empresa start-up de base tecnológica incubada no Polo de Biotecnologia do Rio de Janeiro da Universidade Federal do Rio de Janeiro (UFRJ), de forma a calcular o valor de um projeto de pesquisa específico, tanto pela técnica tradicional de VPL quanto pelo método de Opções Reais, comparando as duas metodologias. Essa avaliação pode ser utilizada para balizar futuras negociações com fundos de venture capital, quando da negociação de um potencial aporte de capital de risco, bem como quando no momento das negociações acerca do licenciamento dos direitos comerciais de determinada droga para uma empresa farmacêutica.

O restante desse artigo está organizado da seguinte forma: na secção 2, apresentamos uma breve revisão de literatura; e, em seguida, os aspectos metodológicos do estudo. Na secção 4, descrevemos o caso estudado e, na secção 5, a aplicação do método proposto e a análise dos resultados. Ao final, apresentamos as conclusões e sugestões para futuras pesquisas.

\section{Revisão da literatura}

$O$ processo de $\mathrm{P} \& \mathrm{D}$ em uma indústria farmacêutica é caracterizado por um longo ciclo de desenvolvimento até o lançamento de uma nova droga no mercado. Além disso, existem significativas incertezas a respeito do custo e do tempo necessário para completar o desenvolvimento, dos resultados da pesquisa e das receitas futuras da comercialização, o que dificulta o processo de análise de investimento e tomada de decisão.
Mesmo diante deste cenário, é necessário que os gestores decidam sobre seus investimentos com grande antecedência (BODE-GREUEL; GREUEL, 2005), considerando as características peculiares deste processo de alocação de recursos. Os investimentos em P\&D voltados para a descoberta de uma nova droga caracterizam-se, de maneira geral, por seu caráter escalonado, sendo os aportes futuros de capital condicionados ao sucesso das fases anteriores de desenvolvimento, o que confere ao gestor importante flexibilidade ao longo do processo de tomada de decisão.

Um projeto de $P \& D$ na área de biotecnologia envolve duas importantes etapas: a descoberta do princípio ativo para desenvolvimento de pesquisas clínicas e a posterior comercialização da droga. A primeira destas etapas é constituída por fases distintas de desenvolvimento antes de ser levado para a aprovação do órgão regulador: screening e desenvolvimento do princípio ativo, testes pré-clínicos e clínicos fase I, II e III (BORISSIOUK; PELI, 2001).

A fase de screening procura identificar estruturas moleculares e/ou princípio ativo que apresente propriedades bioquímicas capazes de combater a doença alvo. Uma das técnicas utilizadas para tal consiste em colocar o agente infeccioso em contato com milhares de compostos bioquímicos, separando aqueles reagentes que apresentarem atividade bioquímica anormal quando exposto a tal ambiente.

A partir da identificação desta molécula, inicia-se a fase de testes pré-clínicos. Por meio de testes farmacológicos, toxicológicos e metabólicos busca-se garantir níveis mínimos de atividade biológica, bem como de segurança da substância testada, antes de se iniciar os testes clínicos em humanos. Caso os testes pré-clínicos apontem para um resultado favorável, prepara-se um documento para ser aprovado pela comissão de ética do órgão regulador, no caso brasileiro a Agência Nacional de Vigilância Sanitária (ANVISA), solicitando autorização para a realização dos testes clínicos em humanos.

A fase I dos testes clínicos tem duração média de 1 a 2 anos e envolve o monitoramento de 20 a 80 voluntários. O principal objetivo é avaliar os níveis de segurança, tolerância e metabolismo da droga quando administrada em seres humanos. Na fase II, também com duração média de 1 a 2 anos, os testes passam a ser realizados diretamente em grupos de 100 a 300 pacientes que apresentem tal doença com o objetivo de verificar a efetividade do tratamento e seus potenciais efeitos colaterais, bem como obter informações sobre a dosagem ideal.

Caso os indicativos das fases I e II sejam promissores, o laboratório dará prosseguimento à fase III dos testes clínicos, mais longa e custosa que as anteriores, podendo durar de 2 a 4 anos, na qual são levantadas evidências estatísticas robustas em 
relação à eficácia e segurança do novo tratamento. Para tanto, são realizados testes duplo-cego em que são comparadas amostras randômicas divididas em grupos de ação e controle, de forma a obter uma comprovação estatística da eficácia terapêutica do tratamento. A partir do resultado positivo apresentado na fase III, todos os relatórios contendo os resultados da pesquisa são enviados para análise pela ANVISA. A decisão do órgão pode ser de aprovação, rejeição ou extensão das pesquisas, caso permaneça alguma dúvida. Caso obtenha a aprovação do órgão regulador, o laboratório necessita ainda incorrer em significativos gastos de divulgação e força de venda para ter sucesso na fase de comercialização da droga.

Um projeto de P\&D está sujeito a duas fontes de risco distintas: o risco tecnológico e o risco de mercado. O primeiro é endógeno e é resolvido à medida que os sucessivos investimentos são realizados ao longo das fases da pesquisa (CHILDS; TRIANTIS, 1999; SCHWARTZ, 2004). O segundo é relacionado às incertezas futuras em relação a sua comercialização. Com relação ao risco tecnológico, Schwartz (2004) destaca que, além da significativa possibilidade de descontinuidade da pesquisa, um projeto de $\mathrm{P} \& \mathrm{D}$ está sujeito a grandes incertezas em relação a seus custos de desenvolvimento. No que tange ao risco de mercado, um projeto de $\mathrm{P} \& \mathrm{D}$ estará sujeito à incerteza do preço futuro praticado e da demanda por determinada aplicação. Investimentos em P\&D devem, portanto, ser compreendidos como um conjunto de opções compostas do tipo arco-íris, sujeitas a diversas fontes de incerteza, representando flexibilidades que agregam significante valor ao projeto de $\mathrm{P} \& \mathrm{D}$.

Segundo Newton, Paxson e Widdicks (2004), as opções de escalonamento dos investimentos acrescentam valor ao projeto, uma vez que permitem a avaliação de sua viabilidade técnica e econômica a cada estágio do programa, permitindo uma reavaliação à medida que novas informações cheguem e resolvam determinada incerteza técnica (CHILDS; TRIANTIS, 1999). Desta forma, o gestor pode limitar os gastos com um programa fadado ao fracasso, reduzindo o prejuízo e redirecionando seus recursos para outros projetos mais promissores.

Intimamente ligada à característica de escalonamento dos investimentos, a possibilidade de licenciar um projeto pouco promissor, dada a piora do cenário econômico, por exemplo, confere à empresa uma opção de abandono, cujo valor será tão maior quanto mais avançada a pesquisa estiver em sua fase de testes e quanto maior for sua probabilidade de sucesso (BORISSIOUK; PELI, 2001). A existência de uma patente também ajudará a elevar o valor econômico desta opção de saída, uma vez que se traduz em uma barreira de entrada a potenciais concorrentes.
Borissiouk e Peli (2001) notam que a opção de espera adiciona valor em projetos de $\mathrm{P} \& \mathrm{D}$, uma vez que as incertezas tecnológicas não são resolvidas apenas com o passar do tempo, mas com o aprendizado adquirido ao longo dos estágios da pesquisa. Os mesmos autores também defendem que um potencial ganho pela espera da redução no nível de volatilidade do mercado acaba sendo completamente dominado pelas vantagens obtidas pelo first mover (NEWTON; PAXSON; WIDDICKS, 2004), impelindo as empresas a acelerarem seus esforços de pesquisa. De forma semelhante, as opções de troca, representadas por substituição de insumos e mudança no mix de produtos, não adicionam valor à atividade de $P \& D$, uma vez que mudanças na composição de determinado medicamento devem ser autorizadas pela agência reguladora, envolvendo novos riscos (BORISSIOUK; PELI, 2001). Por fim, externalidades que porventura surjam ao longo do programa de $\mathrm{P} \& \mathrm{D}$ primário podem vir a inaugurar novas linhas de estudo e potenciais aplicações derivadas a partir da pesquisa inicial - growth options - (HO; LIU, 2003), acrescentando valor ao projeto original, seja pelo acréscimo de uma potencial fonte de receita (LOCH; BODE-GREUEL, 2001), seja pela redução dos riscos de desenvolvimento das novas aplicações.

Cossin, Leleux e Saliasi (2002) enfatizam que, em um primeiro momento, a extrema inabilidade dos analistas em avaliarem uma empresa start-up, altamente inovadora e orientada ao rápido crescimento, poderia se traduzir em paralisia deste mercado de risco, afetando, consequentemente, sua liquidez. Contudo, esta hipótese não se verifica na prática. A indústria, como um todo, tem experimentado um crescimento anual consistente com investimentos acumulados de aproximadamente USD 466 bilhões desde 1970, segundo a National Venture Capital Association (2009). Para responder a este aparente paradoxo, o trabalho de Sahlman (1990) apresenta indícios de que os investidores de risco lidam com as incertezas da avaliação financeira por meio da atuação ativa no desenvolvimento do negócio e no desenho de cláusulas contratuais que favoreçam a relação risco-retorno, e não dedicando horas aprimorando seus modelos ou buscando melhores estimativas.

Os instrumentos contratuais mais usados por esta indústria, guiando o relacionamento entre empreendedor e fundos de venture capital, estabelecem aportes de recursos escalonados, sujeitos à obtenção de marcos de sucesso, além de garantir preferência de liquidez e proteção antidiluição, em caso de novos aportes (SAHLMAN, 1990).

As análises realizadas neste trabalho abordaram apenas um projeto individual, chamado de Projeto Droga Natural, considerando como flexibilidade gerencial, do lado da empresa start up, apenas a possibilidade de interrupção do projeto devido ao 
insucesso em determinada fase da pesquisa. Já sob a ótica da indústria farmacêutica, as opções modeladas foram relativas à suspensão das vendas do novo medicamento, caso não seja bem aceito no mercado e seu crescimento seja abaixo do esperado.

\section{Metodologia}

A avaliação das opções reais foi realizada a partir do modelo binomial discreto proposto por Cox, Ross e Rubinstein (1979), utilizando como ferramenta de apoio o software DPL $®$, que auxiliou na modelagem da árvore de decisão do projeto. A preferência pela modelagem matemática em tempo discreto justifica-se pela própria dinâmica dos investimentos em $\mathrm{P} \& \mathrm{D}$, nos quais novas informações relevantes, em geral, chegam de tempos em tempos, coincidindo com os momentos de decisão sobre a continuidade ou não da pesquisa (BORISSIOUK; PELI, 2001; LOCH; BODE-GREUEL, 2001).

Primeiramente, determinou-se o VPL estático do projeto a partir das técnicas tradicionais de avaliação, servindo este valor como caso base. Para esta etapa, foi necessário definir, além das premissas operacionais, a estrutura de capital e o custo de oportunidade das fontes de financiamento, a fim de calcularmos o custo médio ponderado de capital (WACC) do empreendimento.

Num segundo momento, modelaram-se as incertezas do problema. As de cunho tecnológico foram tratadas de forma probabilística a partir da visão da empresa BioNatural em relação às expectativas de sucesso e fracasso de cada fase da pesquisa. Já as incertezas de mercado, representadas pela evolução da participação de mercado da nova droga, foram modeladas segundo um processo de difusão estocástico geométrico browniano (MGB).

Optamos por modelar diretamente a incerteza a respeito da futura participação de mercado da nova droga, por acreditar ser a variável de maior impacto direto sobre o valor do projeto e por ser, na prática gerencial, o principal direcionador da decisão sobre a manutenção ou não de uma nova droga no portfólio de vendas de uma empresa farmacêutica. Assumimos que o seu processo de difusão é representado pela Equação 1:

$$
d M=\alpha M d t+\sigma M d z
$$

em que: $M=$ participação de mercado no instante $t ; \alpha=$ taxa de crescimento anual da participação de mercado (drift); $\sigma=$ volatilidade da participação de mercado; $d z=\varepsilon \sqrt{d t}$ representa o incremento de Wiener padrão e $\varepsilon \approx \mathrm{N}(0,1)$.

$\mathrm{O}$ apreçamento da opção é feito utilizando-se a medida neutra a risco, em que é deduzido o prêmio de risco do termo de drift do MGB, permitindo que os fluxos de caixa sejam descontados a valor presente pela taxa livre de risco da economia. Em se tratando de ativos de mercado, este prêmio de risco é uma medida conhecida e estabelecida pelos agentes econômicos de acordo com sua percepção e grau de aversão ao risco. Contudo, para ativos cujos mercados são incompletos, é necessário recorrermos a métodos indiretos para o cálculo do prêmio de risco da variável em questão, de forma a determinarmos o valor do termo de tendência (drift) neutro a risco.

Hull (2006) propõe uma aproximação para o cálculo do prêmio de risco de um ativo não negociável no mercado a partir do prêmio de risco do retorno do projeto dado pelo modelo CAPM. Outra forma mais simples e intuitiva é recorrer a um cálculo iterativo valendo-se do fato de que o VPL esperado do projeto descontado ao custo de oportunidade da empresa (WACC) deve ser idêntico ao VPL neutro a risco descontado à taxa livre de risco. Tal condição pode ser expressa algebricamente pela Equação 2 (FREITAS; BRANDÃO, 2010):

$$
\int_{t=1}^{n} E[f(M(t))] e^{-\mu t} d t=\int_{t=1}^{n} E\left[f\left(M_{\phi}(t)\right)\right] e^{-r t} d t
$$

em que, $d M=\alpha M d t+\sigma M d z$ é o processo de difusão verdadeiro da participação de mercado, $d M_{\phi}=(\alpha-\lambda) M_{\phi} d t+\sigma M_{\phi} d z$ é o processo de difusão neutro a risco da participação do mercado, $\lambda$ é o prêmio de risco da participação de mercado $M, \mu \mathrm{e}$ $r$ são, respectivamente, a taxa ajustada ao risco do projeto e a taxa livre de risco, e $E[f(\cdot)]$ é o Fluxo de Caixa Esperado do projeto.

Uma vez determinada a volatilidade e o prêmio de risco implícito do processo estocástico, foram calculados os parâmetros $u$ e $d$, representando, respectivamente, a magnitude dos movimentos de subida e descida emulados pelo ativo base, bem como o valor da probabilidade neutra a risco $p$ e seu complemento $(1-p)$. Por fim, foi construída a árvore de decisão e incorporadas às opções, para permitir a escolha de um conjunto de decisões que venham a maximizar o valor do projeto, obtendo-se um novo resultado para o VPL, chamado de VPL expandido. A diferença entre os resultados das duas avaliações (VPL expandido - VPL estático) representa o valor das Opções Reais presentes no projeto.

Neste estudo, foram utilizados dados da empresa BioNatural e fontes de dados secundárias e públicas; outras formas documentais de informação; entrevistas com o próprio empreendedor para levantar informações de projeções de receita, custos e investimentos previstos para os próximos 20 anos; bem como a identificação das principais fontes de incerteza e flexibilidades gerenciais. Duas entrevistas realizadas junto a um gerente comercial da Merck/ Serono permitiram levantar informações relevantes quanto aos custos operacionais e despesas comerciais relacionados ao lançamento de um novo medicamento. Informações de mercado como a taxa livre de risco e o custo de 
oportunidade do projeto foram obtidas a partir dos sites do Tesouro Direto e do Damodaran, visitados em 23/1/2010.

A maior limitação do modelo diz respeito à escolha do processo estocástico representativo do comportamento do crescimento da participação de mercado do novo medicamento ao longo dos anos de comercialização. Os estudos voltados para a área de novos produtos costumam caracterizar o crescimento das vendas de qualquer novo produto como seguindo uma curva em forma de $S$, possuindo um período inicial de rápido crescimento, seguido de uma desaceleração do ritmo de crescimento, atingindo ao final um estágio de maturação do mercado e da expansão das vendas, passando estas a responderem a um crescimento orgânico, em linha com a indústria e seus competidores.

Optou-se pela escolha do processo estocástico geométrico browniano para representar com mais acurácia apenas o primeiro período de rápida expansão, por aproximar o movimento a uma curva exponencial. Para os períodos subsequentes de desaceleração e estagnação do ritmo de crescimento, acredita-se que a projeção do crescimento das vendas por curvas exponenciais acabe por subestimar a receita futura e consequentemente o valor da empresa.

Embora reconhecida esta limitação, devido ao desconhecimento de outra função que modelasse a curva de crescimento esperado das vendas da nova droga de forma mais acurada, optou-se por utilizar o movimento geométrico browniano como aproximação. Apesar de este processo estocástico não ser a melhor representação do crescimento das vendas, estimou-se que o erro médio represente apenas 3\% da avaliação financeira base do projeto, não implicando em um erro significativo na avaliação do investimento.

\section{Descrição do caso}

A BioNatural tem como foco de atuação o desenvolvimento de pesquisas de novas drogas fitoterápicas a partir de extratos vegetais encontrados na flora brasileira. Detentora de direitos de catalogação e coleta de extratos vegetais oriundos dos mais diversos biomas brasileiros, a BioNatural enfrenta atualmente o desafio de valorar corretamente este ativo intangível. A empresa organizou-se em 1999 a partir de uma parceria com a InterPharma (O nome real foi omitido para preservar a confidencialidade dos dados) por meio do maior contrato de terceirização tecnológica entre uma multinacional farmacêutica e uma empresa de P\&D no País até então. Três anos depois, com a fusão da InterPharma com outra empresa do setor, a mudança de estratégia do novo grupo fez com que a parceria fosse desfeita.

Investidores-anjos e fundos de venture capital, antes sócios desta parceria, também preferiram sair do negócio, induzindo o Sr. Carvalho, dono e empreendedor da BioNatural, a liquidar a empresa BioNatural. A proposta dos acionistas era vender o Banco de Extratos coletados nos últimos 3 anos para a InterPharma a fim de gerar caixa para distribuição entre os sócios investidores. O Sr. Carvalho, no entanto, acreditava que o valor oferecido pelo Banco de Extratos estava muito abaixo do potencial de receita que ele poderia gerar no futuro, em função disso, acabou por comprar a totalidade das ações nas mãos dos investidores. Com isso, a BioNatural deu continuidade aos seus trabalhos de coleta e catalogação de extratos naturais encontrados nos diversos biomas do País, reunindo hoje, em sua extratoteca, um catálogo de cerca de 5.000 espécies vegetais nativas, oficialmente autorizado pelo Conselho de Patrimônio Genético do Ministério do Meio Ambiente, correspondendo a mais de 31.000 amostras vegetais para testes biológicos. $\mathrm{O}$ resultado das pesquisas conduzidas na última década significou para a BioNatural um pipeline de 683 extratos vegetais, com forte atividade direta ou indireta contra seis alvos de interesse médico-farmacêutico: infecções estafilocócicas multirresistentes; doença pulmonar obstrutiva crônica; hepatite C; doença de Chagas; diabetes II; e tuberculose.

A estratégia da empresa para os próximos anos é de levantar mais recursos para financiar as outras linhas de pesquisa e continuar a expansão de seu Banco de Extratos, agregando espécies de outros biomas, como a Floresta Amazônica e o Cerrado brasileiro. Para tanto, é preciso valorar os ativos intangíveis da empresa a fim de balizar o valor das quotas a serem oferecidas aos investidores.

A primeira fase dos estudos, que contempla as etapas de screening e desenvolvimento molecular, embora não exija grandes somas de recursos, se caracteriza por um elevado risco, sendo comum o aporte de seed money por fundos especializados em venture capital ou de angels ou das próprias farmacêuticas, caso tenham grande interesse na linha de pesquisa desenvolvida e, desde o início, queiram garantir opções de preferência em novos aportes.

O financiamento da fase seguinte, de estudos pré-clínicos, exige um aporte mais substancial de recursos envolvendo fundos de venture capital especializados na área de biotecnologia, pois o projeto ainda envolve grande risco. O registro de uma patente, comum durante esta fase, pode reduzir um pouco o grau de incerteza do empreendimento, pois passa a existir uma garantia real, embora de difícil valoração. É comum serem executados, nesta fase, instrumentos contratuais que garantam a opção de preferência de novos aportes, que acabam servindo como um sinal de confiança ao mercado a respeito do sucesso da pesquisa.

A última fase dos testes clínicos é a fase mais intensiva em capital, dada sua longa duração e 
número de pacientes testados. Nesta fase, é comum a busca por uma aliança estratégica entre laboratórios e grandes farmacêuticas, visando o licenciamento da nova droga. Em geral, o acordo envolve um adiantamento up front (fixo) mais a promessa do pagamento de royalties (variável) pelas vendas futuras do novo medicamento. Em contrapartida, o laboratório cede os direitos comerciais da nova droga à farmacêutica que passa a arcar com o custo da fase III dos testes clínicos e de toda a burocracia necessária para a aprovação do novo medicamento junto ao órgão regulador.

No caso da BioNatural, o aspecto singular é o fato de que tanto os testes pré-clínicos quanto os testes clínicos da fase I e II possuem financiamento garantido pelo governo federal a fundo perdido, eliminando a necessidade de capital de risco nestas etapas. Desta forma, a estratégia da empresa contempla apenas o licenciamento da droga para um parceiro estratégico a partir da fase III dos testes clínicos, sem a necessidade de um potencial aporte anterior por parte de um fundo de venture capital.

Embora a empresa possua hoje seis linhas de pesquisa mais avançadas, dada a independência das pesquisas e limitação ao acesso de dados optou-se pela modelagem financeira de um único projeto. $\mathrm{O}$ projeto escolhido visa desenvolver uma aplicação fitoterápica de ação antibiótica e antifúngica, cuja eficácia acredita-se ser comparável aos mais potentes medicamentos existentes no mercado atualmente, apresentando como diferencial o fato de gerar, potencialmente, menor resistência por parte dos micro-organismos patogênicos, dado a origem natural dos componentes químicos da nova droga.

Inicialmente o produto será comercializado sob a forma de pomada tópica servindo para o tratamento de infecções cutâneas, sendo as mais graves, voltadas para pacientes que apresentem úlceras e queimaduras. Embora não consideradas no modelo de avaliação, futuras formas de administração do medicamento, como oral, intramuscular e intravenosa podem vir a ser desenvolvidas após pesquisas incrementais, representando um mercado mais amplo a ser explorado. De acordo com dados internos da empresa, o mercado potencial para este produto é de $\mathrm{R} \$ 125$ milhões, equivalente à venda de 4 milhões de unidades por ano, sendo que a empresa estabeleceu como meta atingir participação de $20 \%$ deste mercado ao final do sétimo ano de comercialização. Depois deste período, as vendas se estabilizariam, passando a crescer de forma orgânica em linha com o resto da categoria.

O cronograma de investimentos para os testes clínicos foi orçado em $\mathrm{R} \$ 5,5$ milhões, sendo $\mathrm{R} \$$ 3,0 milhões garantidos pelo governo federal por um programa de subvenção e direcionados à realização dos testes pré-clínicos e clínicos I e II, com duração prevista de três anos. A outra parcela dos recursos, de R \$ 2,5 milhões, reservada à realização dos testes clínicos da fase III e para homologação do pedido de aprovação da nova droga pela Anvisa, foi planejada para ser financiada por uma farmacêutica interessada em licenciar a nova droga.

A partir destes dados e estimativas das despesas comerciais, administrativas e dos custos operacionais levantados junto ao sócio empreendedor da BioNatural e de especialistas do setor, foram projetados os fluxos de caixa do projeto, expressos em função da participação de mercado e das probabilidades de sucesso de cada fase da pesquisa. Em um primeiro momento, foi feita a avaliação financeira do projeto, utilizando-se a técnica de VPL tradicional, para, em seguida, incorporar o valor das flexibilidades.

O horizonte temporal considerado na análise foi de 14 anos de comercialização, coincidindo com a expiração da patente a ser obtida ao final da fase II dos testes clínicos. O valor residual dos fluxos de caixa livres a partir desta data foi desconsiderado. Todos os números financeiros foram multiplicados por um fator de ajuste, de forma a manter a confidencialidade das informações.

A estimativa da demanda pelo fitoterápico foi considerada como uma fração da participação de mercado do novo medicamento. Assumiu-se que a curva de crescimento apresenta um formato $S$ típico do lançamento de um novo produto, dividida em três fases. Para o primeiro ano de comercialização, considerou-se que a nova droga atingirá $2 \%$ de participação de mercado. A partir deste ponto, modelou-se a participação de mercado da droga com uma fase inicial (fase I) de rápida expansão entre o segundo e quarto ano de comercialização, dobrando a participação de mercado a cada ano, atingindo, ao final deste período, $15 \%$ de participação, seguido de uma segunda fase de desaceleração do ritmo de crescimento, entre o quinto e sétimo ano de comercialização (fase II), chegando a uma participação de mercado de $20 \%$. Por fim, na terceira fase de estabilização do crescimento e manutenção do market share em torno de $20 \%$, a partir do oitavo ano (fase III), o mercado encontrará um novo equilíbrio, e a demanda pela nova droga passará a crescer de forma orgânica, no mesmo ritmo da média de mercado (Figura 1).

Antes de discutirmos as premissas sobre a variável preço, vale destacar uma distorção provocada por esta modelagem no valor do projeto. Conforme verificado na Figura 2, ao modelarmos a segunda fase de crescimento também segundo um MGB (curva superior), cria-se um gap em relação ao formato teórico projetado da curva (área hachurada).

Este gap representa uma subavaliação financeira do projeto devido a uma subcontabilização de receitas durante este período. A fim de reduzir tal erro de cálculo, propomos uma correção do valor, resultado da diferença entre VPL estático cuja demanda segue uma trajetória teórica $S$ (doravante denominado 
$\begin{array}{llll}\text { Fase } 0 & \text { Fase I } & \text { Fase II } & \text { Fase III }\end{array}$

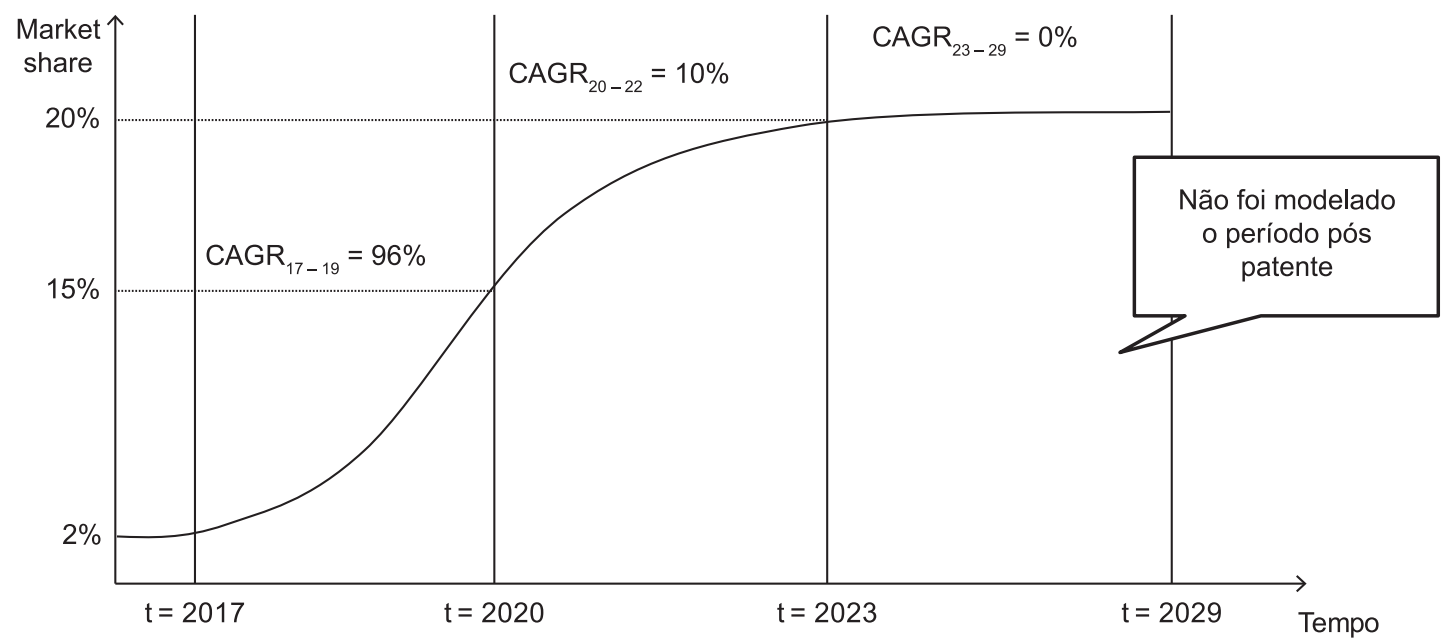

Figura 1. Curva teórica do crescimento esperado do market share do novo medicamento.

$\begin{array}{llll}\text { Fase } 0 & \text { Fase I } & \text { Fase II } & \text { Fase III }\end{array}$

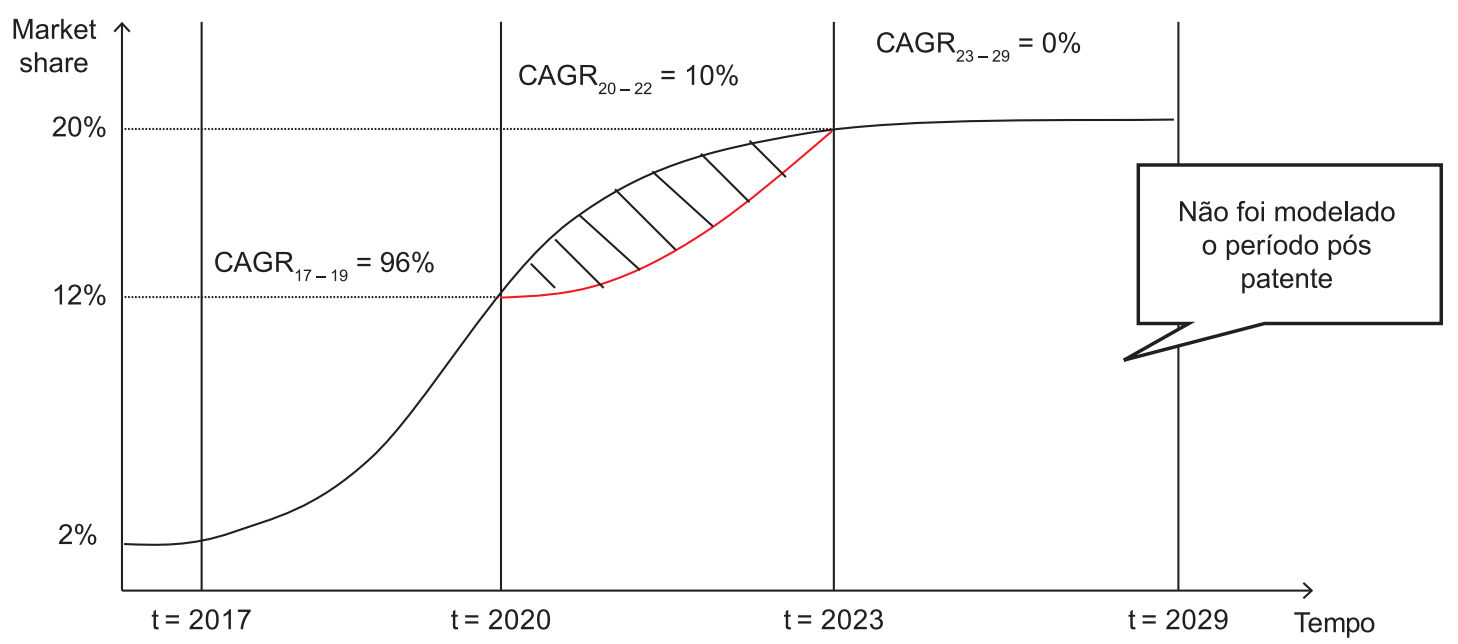

Figura 2. Comparação entre a curva teórica em forma de $S$ e a curva representativa do MGB, representativas do crescimento esperado do market share do novo medicamento.

$V_{P L}{ }^{\mathrm{S}}$ estático) e o VPL estático cuja demanda segue um movimento geométrico browniano (doravante denominado VPL ${ }^{\mathrm{MGB}}$ estático). Matematicamente, a subavaliação pode ser expressa por:

$\Delta$ valor $=$ VPL $^{\mathrm{S}}$ estático $-\mathrm{VPL}^{\mathrm{MGB}}$ estático

$O$ resultado desta diferença revelou uma subavaliação no valor do projeto em torno de $\mathrm{R} \$ 100.000,00$, o equivalente a apenas $3 \%$ do VPL ${ }^{\mathrm{MGB}}$ estático. Desta forma, ao VPL final estimado para o caso base estático, adicionaremos esta quantia como forma de reduzir o erro relacionado à escolha do MGB como processo estocástico representativo do ritmo de crescimento do share de mercado.

Em relação ao preço do produto, dois aspectos particulares do setor devem ser destacados. O primeiro é a forte regulação por parte da Câmara de Regulação do Mercado de Medicamentos (CMED), responsável por fiscalizar e estabelecer as regras que balizam o preço máximo praticado tanto pelos laboratórios fabricantes quanto pela farmácia. O segundo é seu caráter inelástico. Embora não possa ser considerado para todo tipo de medicamento, em geral, a decisão de compra está baseada fortemente na recomendação do profissional da área de saúde, independentemente do aspecto econômico.

Foi adotado um custo de produção unitário de $\mathrm{R} \$ 4,40$, refletindo principalmente o custo da matéria-prima e da embalagem, desconsiderando-se eventuais ganhos de escala futuros. Em relação às despesas operacionais, boa parte dos gastos iniciais está concentrada na divulgação do produto junto 
à comunidade médica, por meio da promoção de eventos comemorativos, distribuição de amostras grátis e realização de visitas comerciais por parte dos representantes laboratoriais. As despesas de marketing para os primeiros 5 anos de comercialização foram estimadas em R \$ 7,5 milhões. Já as despesas de distribuição foram assumidas como variáveis, representando $2 \%$ da receita líquida. As despesas administrativas foram consideradas também como um percentual $6 \%$ da receita líquida.

$\mathrm{O}$ atual projeto encontra-se no início dos testes pré-clínicos, sendo estimados 5 anos de pesquisas até o final da fase III dos testes clínicos. O orçamento total é de R\$ 5,5 milhões, sendo R \$ 3 milhões subvencionados por dinheiro do governo federal. A parcela dos investimentos que deverá, no futuro, ser financiada por uma farmacêutica foi estimada em R \$ 2,5 milhões, principalmente para custear os testes clínicos da fase III.

Dada a preferência do empreendedor em estabelecer uma parceria estratégica que permita a expansão da comercialização de suas drogas para mercados internacionais, optamos por considerar como custo de oportunidade do projeto o WACC de uma subsidiária atuando no Brasil. Para o cálculo do custo de capital próprio, utilizamos um modelo CAPM Global aditivo, ajustado a países emergentes, dada a ausência de empresas abertas do setor farmacêutico no mercado brasileiro. Apesar das possíveis críticas ao modelo, a escolha deste método tem por motivação o fato de que ele é utilizado na prática pela subsidiária brasileira da Merck/Serono, servindo como critério de decisão financeira, sem que a subsidiária tenha qualquer autonomia para questioná-lo ou arbitrar algum outro valor.

Consideramos que a empresa não possui endividamento oneroso, sendo financiada totalmente por capital próprio. Isso é consistente com o padrão do setor farmacêutico mundial que apresenta baixa alavancagem financeira, conforme dados coletados por Damodaran (2009) que revelam uma relação E/ (E+D) de 93\%, em média, no período de 2003-2007 para o setor de biotecnologia. Dessa forma, chegou-se a um WACC, em termos reais e em moeda nacional, de $11 \%$.

Especificadas as premissas operacionais e financeiras, passamos a analisar e quantificar os riscos inerentes ao empreendimento. $\mathrm{O}$ projeto em questão considera duas fontes principais de incertezas. A primeira, de natureza tecnológica, se refere à imprevisibilidade dos resultados da pesquisa em cada fase dos testes laboratoriais. A segunda, originária das forças de mercado, reflete basicamente a incerteza relativa à parcela de mercado conquistada pela nova droga e a natureza da concorrência futura. Embora haja no projeto outras variáveis de caráter incerto, como preço e custo de desenvolvimento da pesquisa, estas foram tratadas de maneira determinística. Maiores referências para a modelagem estocástica dos investimentos em $\mathrm{P} \& \mathrm{D}$ podem ser encontradas em Pindyck (1993).

As incertezas tecnológicas foram tratadas de forma probabilística, como em Loch e Bode-Greuel (2001) e Borissiouk e Peli (2001). Já a incerteza relativa à participação de mercado obtida pela nova droga a cada ano de comercialização foi modelada segundo um processo de difusão geométrico browniano.

Como mencionado anteriormente, considerou-se que, durante o primeiro ano de comercialização, a nova droga atingiria $2 \%$ de participação de mercado. Entre o segundo e o quarto ano de comercialização (fase I), projetou-se um rápido crescimento, dobrando a participação de mercado a cada ano, atingindo, ao final deste período, $15 \%$ de participação, sujeito a uma volatilidade anual estimada em $177 \%$. Entre o quinto e sétimo ano (fase II), projetou-se uma desaceleração do crescimento da participação, alcançando $20 \%$ de participação, sujeita a uma volatilidade em torno de $40 \%$. A partir do oitavo ano (fase III) admitiu-se que o mercado encontrará um novo equilíbrio e que a demanda pela nova droga passa a crescer de forma orgânica, no mesmo ritmo da média de mercado. Desta forma, estimou-se um crescimento nulo da participação de mercado, mas ainda sujeito a um nível de volatilidade na casa de $19 \%$.

Para determinar a volatilidade do ritmo de crescimento, recorremos a um cálculo indireto. A partir da percepção do empreendedor, construímos intervalos de confiança que expressavam os valores mínimos e máximos em torno dos quais a participação de mercado poderia oscilar. Em seguida, traduzimos estas informações em um intervalo de confiança dos valores esperados, mínimo e máximo da taxa de crescimento acumulada. Assumindo uma distribuição triangular para esta variável, estimou-se a volatilidade anualizada para cada uma das três fases de crescimento modeladas, conforme Equação 3:

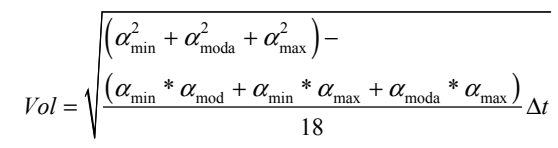

em que,

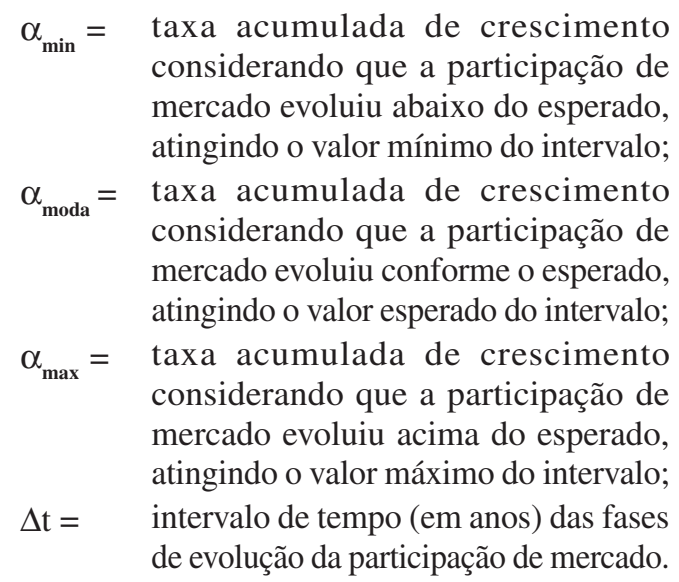


Os resultados estão apresentados nas Tabelas 1 e 2.

\section{Análise dos resultados}

A avaliação financeira sem incorporar as flexibilidades apresentou VPL esperado de R\$ 7,0 milhões. Apesar do VPL esperado positivo, a distribuição dos resultados financeiros foi bastante assimétrica, revelando o elevado risco do projeto. As simulações realizadas utilizando o software Excel revelaram um significativo risco de inviabilidade econômica da pesquisa, ao projetar em apenas $13 \%$ a probabilidade dos retornos estarem acima da taxa mínima de atratividade, conforme pode ser observado na Figura 3.

Tabela 1. Intervalo de variação da participação de mercado a partir das projeções contidas no Plano de Negócios.

\begin{tabular}{ccccc}
\hline Variável & Distribuição & $\mathbf{M}_{\text {min }}$ & $\mathbf{M}_{\text {moda }}$ & $\mathbf{M}_{\mathbf{a x}}$ \\
\hline Part. Mercado 2013 & Triangular & - & $2 \%$ & - \\
Part. Mercado 2016 & Triangular & $0 \%$ & $15 \%$ & $30 \%$ \\
Part. Mercado 2019 & Triangular & $0 \%$ & $20 \%$ & $50 \%$ \\
\hline
\end{tabular}

Depois de modelarmos as incertezas relevantes que afetam o valor do projeto, foi feita a modelagem financeira sob a ótica das Opções Reais. Utilizamos o modelo binomial de Cox, Ross e Rubinstein (1979) para emular o movimento geométrico browniano das três fases de crescimento do ativo-base (participação de mercado). Para fins práticos, foi necessário o cálculo dos parâmetros $u, d$ e $p$, cujos valores podem ser verificados na Tabela 3 e dados por:

$$
u_{i}=e^{\sigma \sqrt{\Delta t}} \quad d_{i}=1 / u \quad p_{i}=\frac{\left(1+\alpha_{i}-\lambda_{i}\right)-d_{i}}{u_{i}-d_{i}}
$$

em que,

$u_{\mathrm{i}}=$ magnitude do movimento de subida na fase $i$;

$d_{\mathrm{i}}=$ magnitude do movimento de descida na fase $i$;

$p_{\mathrm{i}}=$ probabilidade neutra a risco do processo na fase $i$;

$\alpha_{i}=$ taxa de crescimento da participação de mercado na fase $i$;

$\lambda_{i}=$ prêmio de risco relativo à participação de mercado na fase $i$.

Tabela 2. Determinação da volatilidade anualizada da taxa de crescimento da participação de mercado.

\begin{tabular}{cccccc}
\hline Variável & Distribuição & $\alpha_{\text {min }}$ & $\alpha_{\text {moda }}$ & $\alpha_{\max }$ & vol $_{\text {anualizada }}$ \\
\hline Cresc. Acum. 2014-2016 & Triangular & $-100 \%$ & $650 \%$ & $1400 \%$ & $177 \%$ \\
Cresc. Acum. 2017-2019 & Triangular & $-100 \%$ & $33 \%$ & $233 \%$ & $40 \%$ \\
Cresc. Acum. 2020-2030 & Triangular & $-100 \%$ & $0 \%$ & $150 \%$ & $19 \%$ \\
\hline
\end{tabular}

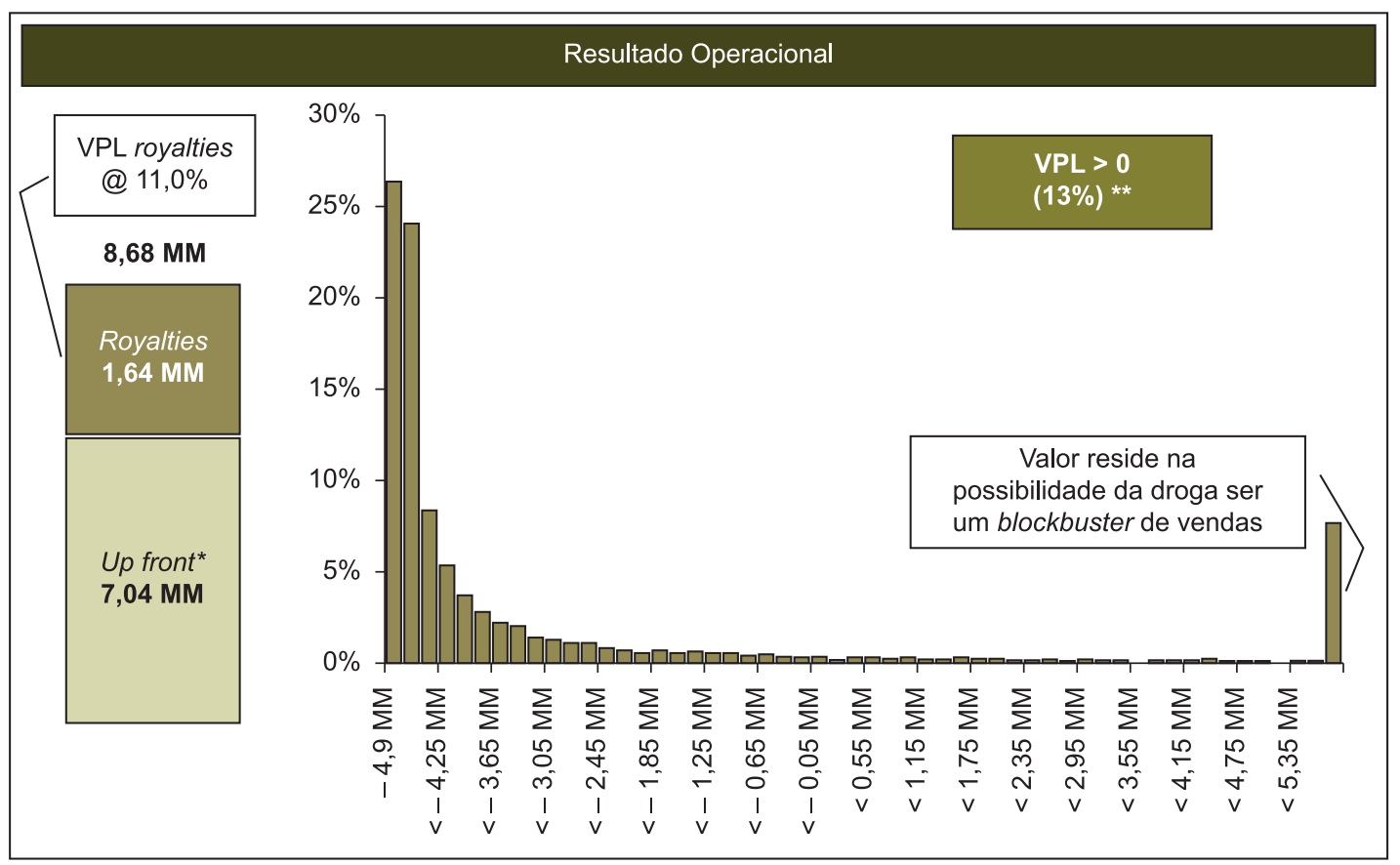

* Incluso os R\$164.000,00 relativos à subavaliação causada pela escolha do MGB como forma funcional da curva de crescimento do share de mercado

** Resultado da simulação de 10.000 iterações

Figura 3. VPL estático e distribuição de probabilidade do VPL. 
A partir destes parâmetros foi modelada a árvore binomial (Figura 4), que emula a curva de crescimento da participação de mercado do novo medicamento.

Para validar o modelo, realizamos a simulação dos resultados sem a introdução das flexibilidades gerenciais (nós de decisão). Para garantir a robustez do modelo, realizamos primeiramente um teste para garantir que o resultado da simulação seria igual ao valor do VPL estático da modelagem financeira realizada em Excel. A comparação dos resultados

Tabela 3. Parâmetros do modelo binomial neutro a risco.

\begin{tabular}{cccccc}
\hline \multicolumn{6}{c}{ Inputs para o modelo binomial de Cox, Ross e } \\
Rubinstein (1979)
\end{tabular}

garantiu que ambos eram equivalentes, assegurando que o modelo binomial estava bem especificado, conforme pode ser observado na Figura 5.

Em seguida, introduziram-se as flexibilidades gerenciais, sob a forma de nós de decisão. As incertezas tecnológicas foram expressas em termos de probabilidade. Para caracterizar a opção de abandono das pesquisas, modelamos um galho de sucesso e fracasso dos testes clínicos da fase III, atribuindo uma probabilidade de $80 \%$ de sucesso da pesquisa ao final da fase. Mesmo em caso de sucesso, a nova droga ainda precisa ganhar a aprovação da ANVISA. O raciocínio é análogo, tendo sido estabelecida uma chance de $95 \%$ de aprovação por parte do órgão regulador.

As incertezas mercadológicas foram expressas em termos estocásticos. Para modelar a incerteza relativa à evolução do crescimento das vendas da nova droga, inserimos um nó de decisão a cada ano

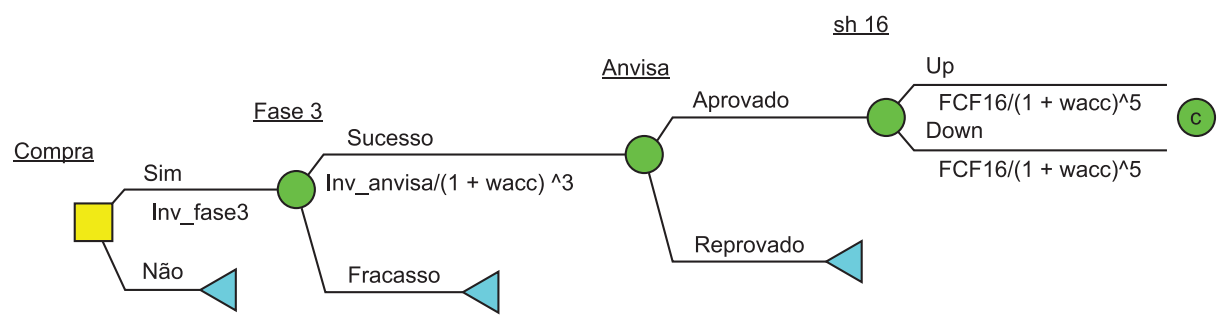

$\underline{\operatorname{sh} 17}$

$\underline{\operatorname{sh} 18}$

$\underline{\operatorname{sh} 19}$

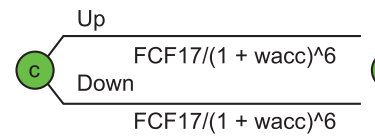

$\underline{\operatorname{sh} 20}$

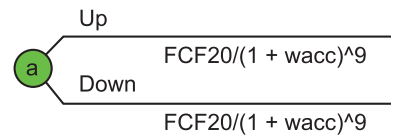

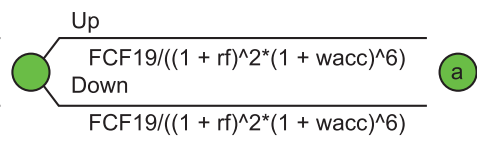

$\underline{\operatorname{sh} 22}$

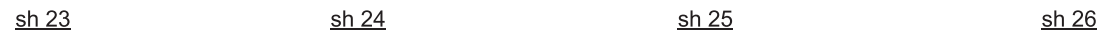

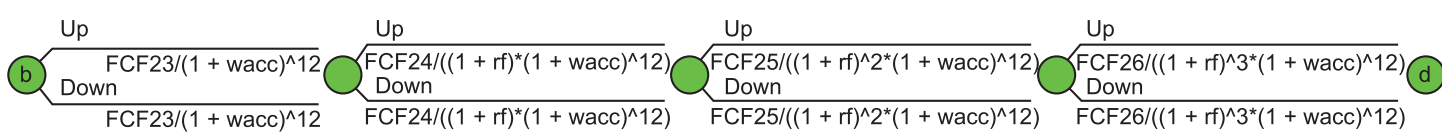

$$
\begin{aligned}
& \underline{\operatorname{sh} 27} \underline{\text { sh } 28} \quad \underline{\text { sh } 29}
\end{aligned}
$$

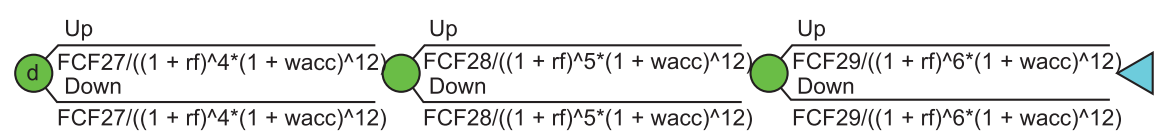

Figura 4. Modelagem da malha binomial relativa à evolução da participação de mercado do novo medicamento relativa ao caso base, sem a introdução dos nós de decisão. 
de comercialização, significando que, em caso de fracasso das vendas e baixa perspectiva de melhora do cenário, a decisão a ser tomada pelo gestor será abandonar o negócio e minimizar as perdas previstas. Em caso de fracasso da política comercial, o modelo apontará para a decisão de desinvestimento, possibilitando à farmacêutica uma economia de gastos, principalmente os relacionados às despesas comerciais; caso contrário, a decisão será continuar a comercializar a nova droga.

A decisão de abandono foi baseada em uma análise comparativa, ano a ano, entre o VPL obtido em caso de manutenção das vendas da nova droga e o VPL obtido em caso de abandono da comercialização. A decisão de abandonar ou não dependerá exclusivamente de qual alternativa apresentar o maior VPL esperado. Considerando a possibilidade de abandono da comercialização ao longo dos 14 anos sob a proteção da patente, podemos verificar que há um incremento da ordem de $13 \%$ ou R $\$ 915$ mil em relação ao VPL estático ajustado. A Figura 6 ilustra a adição de valor das opções reais.

É importante destacar que esta adição de valor significa o reconhecimento de que os gestores de uma farmacêutica serão ativos ao se depararem com um cenário de fracasso comercial do novo medicamento e consequente destruição de valor, optando pela decisão de descontinuidade da produção.

Para conhecermos a probabilidade de exercício de cada opção de abandono, simulamos novamente a malha binomial, desta vez considerando o risco existente no fluxo e na taxa de desconto. Utilizamos, no lugar da taxa de crescimento livre de risco, a verdadeira taxa de crescimento (CAGR) para cada fase. No lugar da taxa livre de risco, descontaremos os fluxos de caixa pelo próprio WACC do projeto. Vale destacar que este procedimento, embora permita conhecer os verdadeiros valores das probabilidades de exercício das opções reais, não valora corretamente as opções, devendo o resultado do modelo ser desconsiderado para qualquer análise.

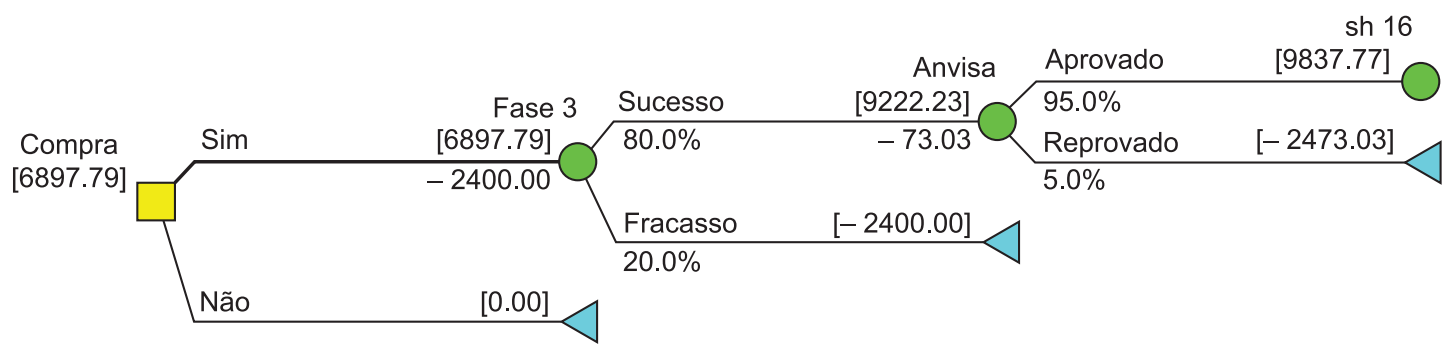

Figura 5. Resultado do modelo binomial relativo ao caso base, sem a introdução dos nós de decisão (VPL estático não ajustado).

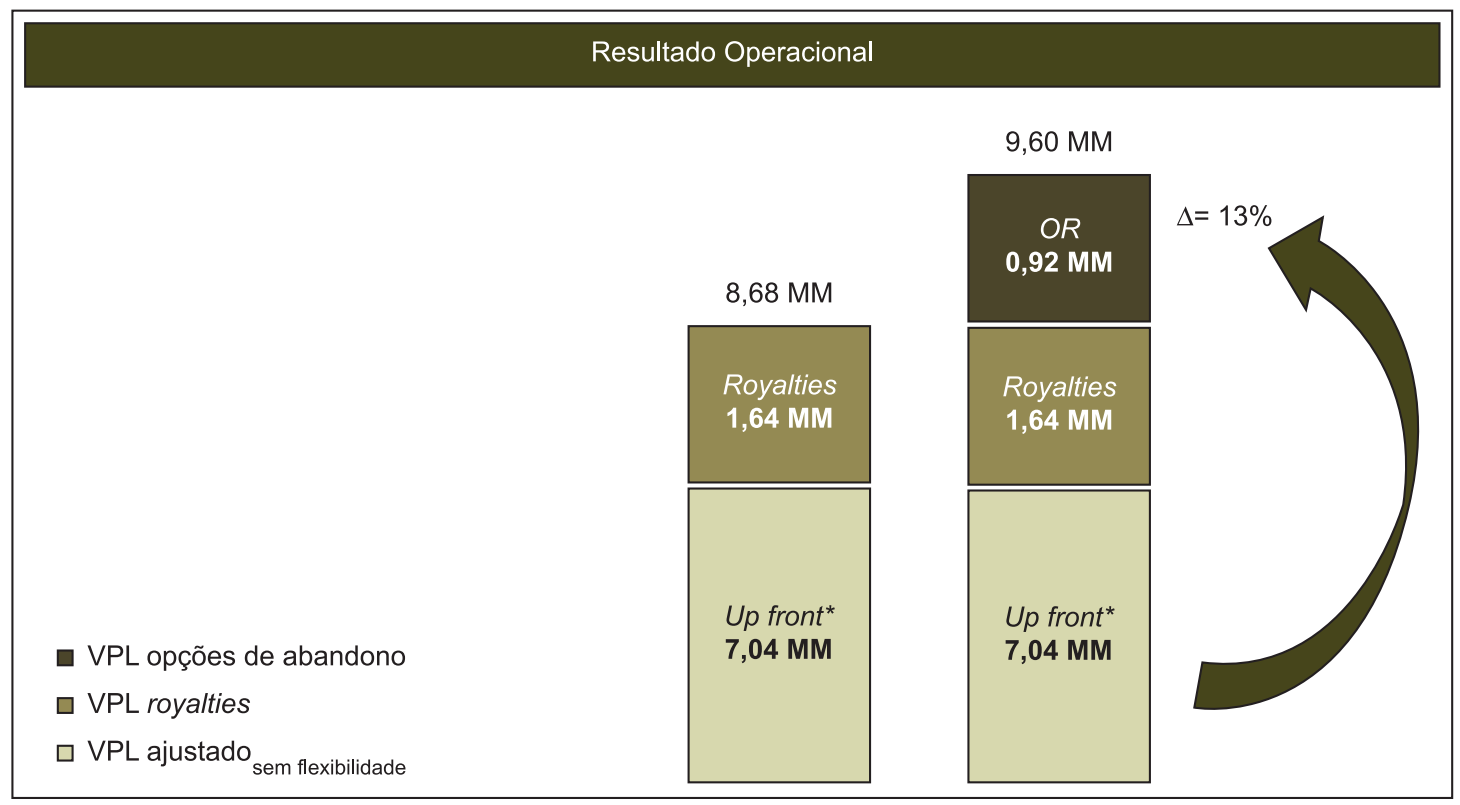

* Incluso os $\mathrm{R} \$ 164.000,00$ relativos à subavaliação causada pela escolha do MGB como forma funcional da curva de crescimento do share de mercado

Figura 6. Valor presente das Opções Reais de abandono. 
Tabela 4. Probabilidades de exercício das opções de abandono, condicionadas ao sucesso da fase III dos testes clínicos e à aprovação da ANVISA.

\begin{tabular}{cccc}
\hline Opção & $\begin{array}{c}\text { \% } \\
\text { Continuidade* }\end{array}$ & $\begin{array}{c}\% \\
\text { Abandono* }\end{array}$ & $\begin{array}{c}\text { \% Não } \\
\text { Ocorrência* }\end{array}$ \\
\hline Ab16 & 100,0 & 0,0 & 0,0 \\
\hline Ab17 & 53,0 & 0,0 & 0,0 \\
Ab18 & 23,4 & 47,0 & 0,0 \\
Ab19 & 23,4 & 29,6 & 47,0 \\
\hline Ab20 & 23,4 & 0,0 & 76,6 \\
Ab21 & 23,4 & 0,0 & 76,6 \\
Ab22 & 23,4 & 0,0 & 76,6 \\
Ab23 & 23,4 & 0,0 & 76,6 \\
Ab24 & 23,4 & 0,0 & 76,6 \\
Ab25 & 23,4 & 0,0 & 76,6 \\
Ab26 & 23,4 & 0,0 & 76,6 \\
Ab27 & 23,3 & 0,1 & 76,7 \\
Ab28 & 23,0 & 0,3 & 76,7 \\
\hline Probabis
\end{tabular}

*Probabilidades condicionadas à aprovação da droga junto à Anvisa.

O resultado do exercício das opções pode ser verificado na Tabela 4. Cabe ressaltar que tais probabilidades representam a possibilidade de exercício ou não de determinada opção a priori, quando do momento do licenciamento da nova droga $(t=2011)$. A coluna "\% Não Ocorrência" representa, portanto, a probabilidade de não acontecer a comercialização da droga em determinado ano. Já as colunas "\% Abandono" e "\% Continuidade" representam, respectivamente, a probabilidade de abandono e continuidade da comercialização.

O modelo revela, como era de se esperar, elevada probabilidade de exercício das opções de abandono durante os anos iniciais de comercialização. De acordo com os resultados, a nova droga apresenta uma probabilidade de vir a ser economicamente viável de pouco mais de $13 \%$.

\section{Considerações finais}

O objetivo deste artigo foi investigar a possível contribuição da metodologia de Opções Reais na avaliação de projetos de P\&D na área de biotecnologia, tentando mensurar se a avaliação tradicional, por meio do VPL estático, subavaliava os projetos, ao desconsiderar a capacidade dos gestores de uma farmacêutica em cessar os gastos relativos ao marketing e retirar de linha o novo medicamento em caso de baixa aceitação do mercado.

As análises realizadas revelaram que não considerar o valor das opções de abandono da comercialização subavaliava o valor a ser negociado como up front do licenciamento da nova droga em $13 \%$, o equivalente a R\$ 915 mil, valor bastante significativo para o empreendedor que utilizará estes recursos para subsidiar novas pesquisas. Verificou-se também que as opções de abandono possuem mais valor nos períodos iniciais do lançamento da nova droga no mercado, exatamente o momento de maior incerteza, em que o gestor pode decidir retirar o novo medicamento de linha no caso de fracasso comercial e baixa perspectiva de alteração do cenário. Esta possibilidade reduz o risco global do empreendimento, adicionando valor quando comparada à abordagem estática do VPL, que considera o investimento como um gasto afundado, sujeito ao mesmo risco ao longo de todos os anos.

Em teoria, a abordagem das Opções Reais se mostra mais robusta e complementa as imperfeições da teoria do Valor Presente Líquido. Entretanto, pudemos perceber que a disposição de um comprador em elevar seu preço de reserva e, consequentemente, sua disposição a pagar por algumas opções embutidas em um determinado empreendimento pode não se concretizar, devido ao receio de estar se pagando por uma flexibilidade cujo efetivo exercício pode estar limitado por diversos aspectos políticos e gerenciais.

Os resultados obtidos neste trabalho sugerem que a metodologia das Opções Reais possui algumas vantagens em relação à análise pelo Fluxo de Caixa Descontado tradicional no caso de empresas start-ups na área de biotecnologia como no caso analisado neste trabalho. $\mathrm{O}$ método do FCD estático ignora o valor da opção de escalonar os investimentos e de abandonar o empreendimento no caso de insucesso, o que leva à subavaliação do negócio.

Como sugestão de pesquisas futuras, sugerimos tanto a modelagem dos custos de desenvolvimento de forma estocástica, dada a grande variabilidade do tempo necessário para o desenvolvimento das pesquisas, quanto a consideração de opções de crescimento, relacionadas a novas aplicações descobertas a partir da pesquisa original. Outra área de estudos a ser explorada no futuro está relacionada à modelagem de cláusulas contratuais de antidiluição e preferência no exercício de novos aportes, à medida que as pesquisas de $P \& D$ vão se mostrando promissoras. A modelagem do relacionamento entre fundos de venture capital e empreendedores, via estes instrumentos jurídicos, impacta o valor dos investimentos e, portanto, deve ser considerada na hora da avaliação dos projetos.

\section{Agradecimentos}

Os autores agradecem o apoio do CNPq para este artigo e Bio-Rio - Pólo de Biotecnologia do Rio de Janeiro.

\section{Referências}

AMRAM, M.; KULATILAKA, N. Real Options - Managing Strategic Investment in an Uncertain World. Boston: Harvard Business School Press, 1999. 
BODE-GREUEL, K. M.; GREUEL, J. M. Determining the value of drug development candidates and technology platforms. Journal of Commercial Biotechnology, v. 11 , p. $155-170,2005$. http://dx.doi.org/10.1057/ palgrave.jcb.3040113

BORISSIOUK, O.; PELI, J. Real Option Approach to R\&D Project Valuation: Case Study at Serono International S.A. The Financier, v. 8, n. 1-4, p. 7-71, 2001.

CHILDS, P. D.; TRIANTIS, A. J. Dynamic R\&D Investment Policies. Management Science, v. 45, n. 10, p. 13591377, 1999. http://dx.doi.org/10.1287/mnsc.45.10.1359

COPELAND, T.; ANTIKAROV, V. Opções Reais. São Paulo: Editora Campus, 2002.

COSSIN, D.; LELEUX, B.; SALIASI, E. Understanding the Economic Value of Legal Covenants in Investment Contracts: A Real-Options Approach to Venture Equity Contracts. SSRN eLibrary, 2002.

COX, J. C.; ROSS, S. A.; RUBINSTEIN, M. Option pricing: A simplified approach. Journal of Financial Economics, v. 7, n. 3, p. 229-263, Sept 1979. http:// dx.doi.org/10.1016/0304-405X(79)90015-1

DAMODARAN, A. Damodaran Online. Disponível em: <http://pages.stern.nyu.edu/ adamodar/>. Acesso em: 17 ago. 2009.

DIXIT, A. K.; PINDYCK, R. S. Investment under Uncertainty. Princeton: Princeton University Press, 1994.

FREITAS, A.; BRANDÃO, L. Real Options Valuation of e-learning Projects. International Journal on E-Learning, v. 9, n. 3, p. 363-383, 2010.

HO, S. P.; LIU, L. Y. How to Evaluate and Invest in Emerging A/E/C Technologies under Uncertainty. Journal of Construction Engineering and Management, v. 129, n. 1, p. 16-24, 2003. http://dx.doi.org/10.1061/ (ASCE)0733-9364(2003)129:1(16)

HULL, J. C. Options, Futures and Other Derivatives Securities. 6th. ed. Englewood Cliffs: Prentice Hall 2006.

LOCH, C. H.; BODE-GREUEL, K. Evaluating growth options as sources of value for pharmaceutical research projects. R\&D Management, v. 31, n. 2, p. 231-248, 2001. http:// dx.doi.org/10.1111/1467-9310.00212

NATIONAL VENTURE CAPITAL ASSOCIATION. Disponível em: <http://www.nvca.org/>. Acesso em: 10 jul. 2009.

NEWTON, D. P.; PAXSON, D. A.; WIDDICKS, M. Real R\&D options. International Journal of Management Reviews, v. 5, n. 2, p. 113-130, 2004. http://dx.doi. org/10.1111/j.1460-8545.2004.00099.x

PINDYCK, R. S. Investments of Uncertain Cost. Journal of Financial Economics, v. 34, n. 1, p. 53-76, Aug 1993. http://dx.doi.org/10.1016/0304-405X(93)90040-I

SAHLMAN, W. A. The structure and governance of venture-capital organizations. Journal of Financial Economics, v. 27, n. 2, p. 473-521, 1990. http://dx.doi. org/10.1016/0304-405X(90)90065-8

SCHWARTZ, E. S. Patents and R\&D as Real Options. Economic Notes - Review of Banking, Finance and Monetary Economics, v. 33, n. 1, p. 23-54, 2004.

TRIGEORGIS, L. Real options, Managerial Flexibility and Strategy in Resources Allocation. Cambridge: MIT Press, 1996.

VILLIGER, R.; BOGDAN, B. Pitfalls of valuation in biotech. Journal of Commercial Biotechnology, v. 12, p. 175-181, 2006. http://dx.doi.org/10.1057/palgrave. jcb.3040164 